\title{
Protective effect of zoledronic acid on articular cartilage and subchondral bone of rabbits with experimental knee osteoarthritis
}

\author{
GUORONG SHE $^{1 *}$, ZIQIZHOU $^{2 *}$, ZHENGANG ZHA $^{3}$, FEI WANG $^{4}$ and XIAOTING PAN ${ }^{5}$ \\ ${ }^{1}$ Department of Orthopedics, The First Affiliated Hospital of Jinan University, Guangzhou, Guangdong 510630; \\ ${ }^{2}$ Department of Radiation Therapy, Peking Union Medical College Hospital (East), Beijing 100000; \\ Departments of ${ }^{3}$ Bone and Joint Surgery, ${ }^{4}$ Medical Imaging Center and ${ }^{5}$ Pathology, \\ The First Affiliated Hospital of Jinan University, Guangzhou, Guangdong 510630, P.R. China
}

Received March 5, 2016; Accepted May 25, 2017

DOI: $10.3892 / \mathrm{etm} .2017 .5135$

\begin{abstract}
Subchondral bone reabsorption and remodeling are responsible for the initiation and progression of osteoarthritis (OA). Zoledronic acid (ZOL), a third-generation bisphosphonate (BIS), is an inhibitor of bone reabsorption. However, the intervention effect of ZOL on OA has not been fully characterized and remains to be directly demonstrated in animal experiments. The present study examined the microscopic and macroscopic changes in the anterior cruciate ligament transection (ACLT) model of OA in rabbits and evaluated the effects of ZOL on cartilage degeneration and subchondral bone loss. A total of 32 New Zealand white rabbits were randomly divided into four groups: High-, medium- and low-dose ZOL groups, which received an intravenous injection of 250 , 50 and $10 \mu \mathrm{g} / \mathrm{kg} \mathrm{ZOL}$, respectively, after modeling, as well as an untreated group. The bone mineral density (BMD) of the knee joint was evaluated by dual-energy X-ray absorptiometry scanning immediately after modeling and at 4 and 8 weeks. At week 8, quantitative measurement of cartilage was performed by a specialized magnetic resonance imaging (MRI) technique, including three-dimensional fat-suppressed spoil gradient-recalled sequence and T2 mapping. The rabbits were sacrificed by air embolism after anesthesia and both knee joints were harvested and evaluated by general and histological observation. Toluidine blue and hematoxylin and eosin staining were used to assess histological changes in the articular cartilage. Quantitative analysis of cartilage histopathology was performed according to the Mankin scoring system. The BMD of ACLT joints dropped after modeling,
\end{abstract}

Correspondence to: DrZhengang Zha,Department of Bone and Joint Surgery, The First Affiliated Hospital of Jinan University, 613 West Huangpu Avenue, Guangzhou, Guangdong 510630, P.R. China E-mail: zhzgg@vip.163.com

*Contributed equally

Key words: zoledronic acid, osteoarthritis, articular cartilage, subchondral bone, bone mineral density which was effectively suppressed by ZOL at the high and medium dose but not the low dose. MRI scans demonstrated that in the untreated group, articular cartilages on ACLT knees were thinner than those on normal knees. The high dose of ZOL preserved the cartilage tissue thickness more efficiently than the medium and low doses. Observation of specimens and pathological slices revealed that the articular cartilage degeneration in the high-dose ZOL group was lightest, while that in the medium- and low-dose ZOL group was moderate, and the untreated group exhibited the most severe defect. The untreated group had the highest Mankin score, whereas the high-dose ZOL group had the lowest score. In conclusion, ZOL increased the subchondral bone density, improved the microstructure and reduced the degeneration of articular cartilage in OA according to morphological as well as quantitative observation. ZOL exerted significant chondroprotective effects in a dose-dependent manner. A favorable chondroprotective effect was induced at the dose of $250 \mu \mathrm{g} / \mathrm{kg}$. ZOL may represent a novel promising drug to complement the treatment of OA.

\section{Introduction}

Osteoarthritis (OA) is a chronic whole-organ disease, which is characterized by hyaline articular cartilage degeneration, bone sclerosis and osteophyte formation. It also involves the periarticular muscle, ligaments, synovium, the neurosensory system and eventually the bone. It is increasingly accepted that the subchondral bone has an important role in the pathogenesis of OA, while the significance of changes inside the subchondral bone remain controversial (1).

Emerging evidence supported that bone remodeling is responsible for the progression of OA (2-4). Early OA is associated with increased bone reabsorption, indicating that increases in bone reabsorption are partly responsible for initiating cartilage destruction under stressful conditions $(3,5)$. This pathological progression increases the possibility that early application of a bone reabsorption inhibitor may retard the progressive loss of articular cartilage.

Bisphosphonates (BIS), including alendronate, ibandronate and risedronate, are potent inhibitors of osteoclastic bone reabsorption and have been used in the clinic for the treatment of osteoporosis and for the treatment/prevention 
of skeletal-associated events in multiple myeloma as well as breast and prostate cancer patients (6-8). While majority of studies demonstrated that BIS are effective in inhibiting the progression of OA via increasing the periarticular bone volume and bone mineral concentration $(3,5)$, BIs remain under debate, as other emerging studies suggested that BIS aggravates cartilage degeneration in spite of enhancing subchondral bone volume and thickness (9). For decades, firstand second-generation BIS have been the focus of major basic or clinical research. Zoledronic acid (ZOL), the representative of the third generation of BIS, has a comparatively higher efficacy and is commonly used in the treatment of osteoporosis, particularly in post-menopausal women, but its mechanism of action remains elusive (10). As a novel promising and potent BIS, the effect and underlying mechanism of ZOL deserve to be elucidated. The present study established an OA model in the knees of rabbits (KOA model) through anterior cruciate ligament transaction (ACLT). Intravenous injection of a high, medium or low dose of ZOL was applied as an intervention. It was assessed whether, by preventing increased subchondral bone reabsorption and remodeling, ZOL treatment suppressed OA progression. It was assumed that ZOL may intervene with cartilage degeneration in the early stage of OA. The purpose of the present study was to determine the chondroprotective effect of ZOL.

\section{Materials and methods}

Animals. A total of 32 New Zealand White rabbits (male; age, 5-7 months; weight, 2-3 kg) were provided by the Laboratory Animal Center of the Academy of Guangdong Province (Guangzhou, China). They were housed under constant laboratory conditions with free access to food and distilled water and a natural light-dark cycle. The present study was approved by the Laboratory Animal Ethics Committee of Jinan University (Guangzhou, China; no. 2008A001).

Drugs and reagents. The following drugs and reagents were used in the present study: Injection of ZOL (Novartis International AG, Basel, Switzerland), injection of sumianxin II (Veterinary Institute of Medical Science Military Academy, Changchun, China), pentobarbital sodium (Shanghai Chemical Reagent Factory, Shanghai, China), toluidine blue (Amresco, Inc., Framingham, MA, USA), eosin hematoxylin, formaldehyde, xylene, anhydrous ethanol (all from Guangzhou Chemical Reagent Factory, Guangzhou, China) and chloral hydrate (Dalian Meilun factory, Dalian, China).

The dose of ZOL administered to the animals was calculated by the conversion formula of surface area based on that for humans. It was defined by the following equation: Dose for rabbits $(2.0 \mathrm{~kg})=$ dose for humans $(60 \mathrm{~kg}) \times 0.07]^{2 / 3}=$ $(5,000 \times 0.07)^{2 / 3}=50 \mu \mathrm{g}$. Therefore, a high dose of ZOL $(\mathrm{ZH})$, a medium dose of ZOL (ZM) and a low dose of ZOL (ZL) group were established, which were administered ZOL at doses of 250,50 and $10 \mu \mathrm{g} / \mathrm{kg}$, respectively.

Laboratory apparatus. Dual-energy X-ray absorptiometry (DXA) was performed using the Lunar Prodigy instrument (GE Healthcare, Little Chalfont, UK). Furthermore, a Signa1.5T HD Superconducting magnetic resonance imaging
(MRI) scanner (GE Healthcare) and an IX 71 inverted phase contrast microscope (Olympus Co., Ltd., Tokyo, Japan) were used.

Study design. After the rabbits had acclimatized to the new environment for 2 weeks, the KOA model was established by ACLT. The animals underwent ACLT on the left hind knee (ACLT knee) and a sham operation on the right hind knee (sham knee), where the knee underwent mostly similar operation steps except that the anterior cruciate ligament was left untouched. The procedure of the surgery was followed strictly as described previously (11). In addition, 3 days prior to and after surgery, 400,000 units penicillin were intramuscularly injected twice a day to prevent infection. The rabbits were allowed full weight-bearing post-operatively and then randomized into 4 groups: $\mathrm{ZH}$ group (group $\mathrm{A} ; \mathrm{n}=8$ ), ZM group (group $B ; n=8), Z L$ group (group $C ; n=8$ ) and untreated group (group $\mathrm{D} ; \mathrm{n}=8$ ), which received intravenous injection of ZOL at 250,5010 or $0 \mu \mathrm{g} / \mathrm{kg}$ in saline, respectively, via the ear marginal vein once post-surgery. DXA scanning was performed at 0,4 and 8 weeks after modeling. At the 8th week, MRI was performed and subsequently, all rabbits were sacrificed by air embolism, during which the rabbits were anesthetized by intraperitoneal injection of $10 \%$ chloral hydrate at the dose of $400 \mathrm{mg} / \mathrm{kg}$ and $10 \mathrm{~min}$ later, $30 \mathrm{ml}$ air was injected into the ear margin. Cartilage samples were obtained to prepare sections for hematoxylin and eosin $(H \& E)$ and toluidine blue staining. Histological samples were statistically analyzed using the Mankin scoring system (12) to evaluate the pathological changes of the cartilage.

DXA scanning. DXA analysis was performed at 0,4 and 8 weeks after modeling. Measurements were taken in vivo with the rabbits placed in the prone position under general anesthesia with $0.2 \mathrm{ml} / \mathrm{kg}$ Sumianxin II. DXA analysis was performed with analysis software enCORE 10.50.086 (Lunar Prodigy Scientific Research; GE Healthcare) for animals. The area was scanned between lower the half of the thigh and the upper half of the tibia. Rectangular regions $(20 \times 15 \mathrm{~mm})$ at the center of the knee as well as the medial and lateral condyle of the tibia were assessed to represent the mean bone mineral density (BMD) of the whole knee joint (WKJ). Another 4 square compartments $(2.5 \times 2.5 \mathrm{~mm})$ were selected to assess the subchondral bone density on the most protruding point of the medial and lateral condyle of the femur and lateral condyle of the tibia separately, which were defines as femoral medial condyle (FMC), femoral lateral condyle (FLC), tibial medial condyle (TMC) and tibial lateral condyle (TLC) for explicit recording. The location of compartments was selected as illustrated in Fig. 1. The BMD at 0 week was considered as the baseline parameter. As significant individual differences in BMD were identified, changes in BMD following surgery in the ACLT and sham joints were assessed by comparison with each joint's baseline measurements (internal control) (4).

MRI scanning. At 8 weeks after modeling, rabbits were anaesthetized with $3 \%$ pentobarbital sodium $(30 \mathrm{mg} / \mathrm{kg})$ administered via the ear marginal vein and the two knees were examined simultaneously. All examinations were standardized by using a dedicated device allowing the rabbits to be placed in a supine 

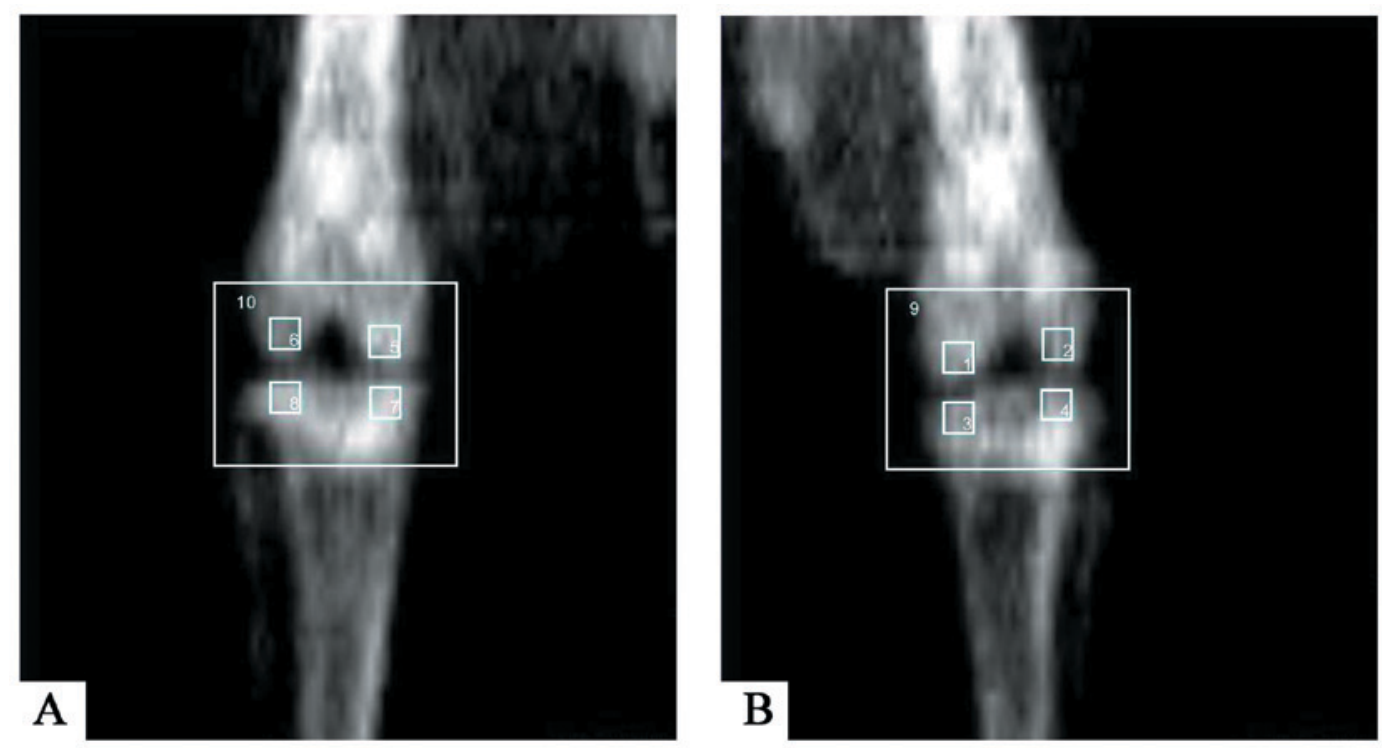

Figure 1. DXA scanning in five compartments of (A) the right knee and (B) the left knee. In total, the bone mineral density of five compartments [femoral medial condyle (left 1 , right 5), femoral lateral condyle (left 2, right 6), tibial medial condyle (left 3, right 7), tibial lateral condyle (left 4, right 8), and whole knee joint of the right (10) and left (9) control was evaluated by DXA]. DXA, dual-energy X-ray absorptiometry.
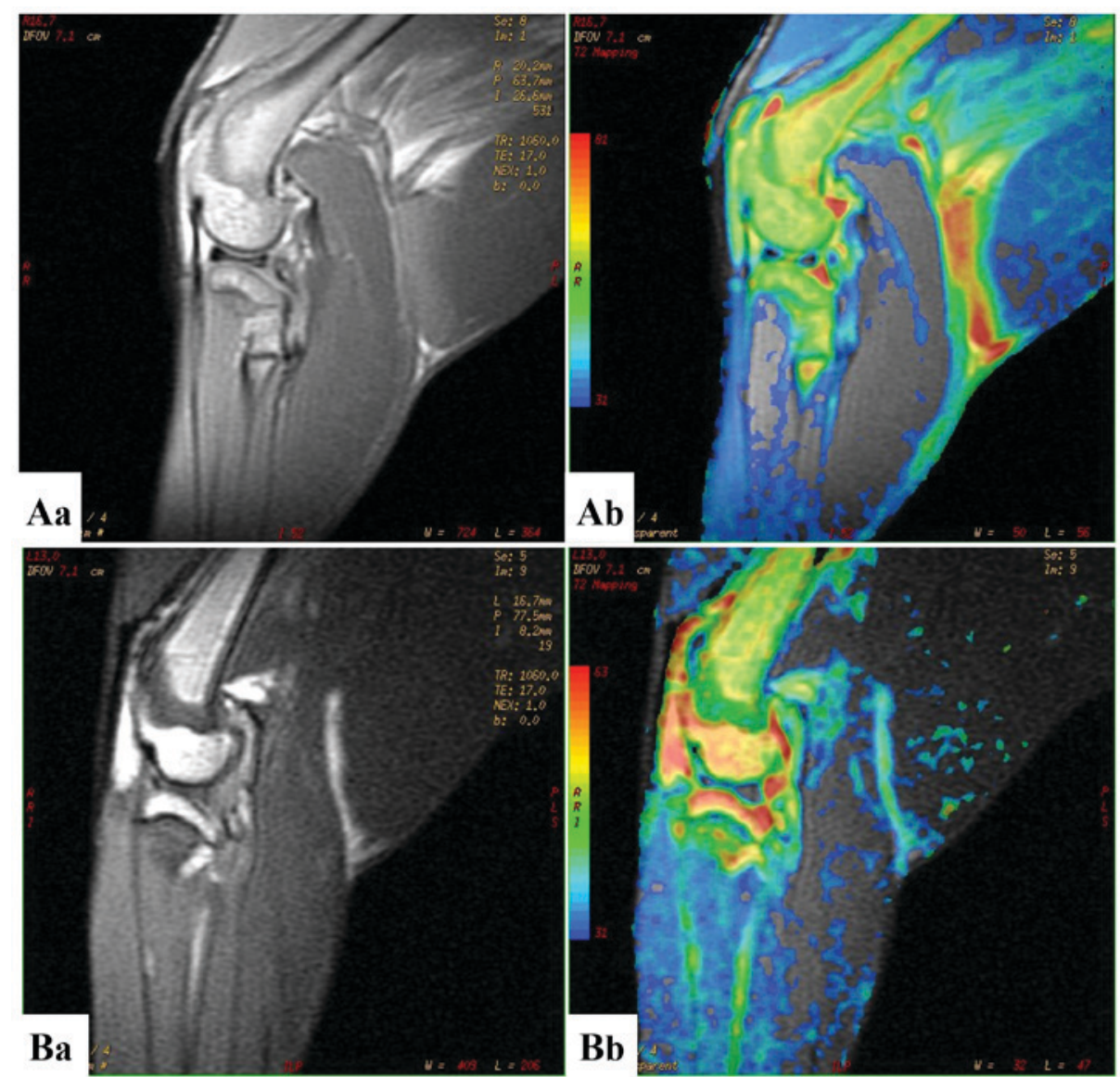

Figure 2. T2 mapping in saggital position of (A) the normal knee joint and (B) the OA joint. (Aa) In the original T2-mapped image, the normal articular cartilage displayed homogeneous hyperintensity and $(\mathrm{Ab})$ in the T2-mapped pseudo-color image, the normal articular cartilage was uniform light green and subchondral bone was yellow-green. (Ba) In the T2-mapped image of the $\mathrm{OA}$ joint, $\mathrm{OA}$ cartilage had a slightly inhomogeneous hyperintensity and (Bb) in the T2-mapped pseudo-color image, OA cartilage was less uniformly green and subchondral bone was orange (B2). OA, osteoarthritis.

position with the leg placed in the scanner at slight flexion. The knee underwent MRI scanning through the saggital position with T2-weighted imaging, three-dimensional fat-suppressed spoil gradient-recalled sequence (3D-FS-SPGR) and T2 mapping (Fig. 2). The images were prospectively analyzed by two independent musculoskeletal MRI radiologists blinded 
to the grouping using the Sun Advantage Workstation 4.2 post-processing software (GE Healthcare). The radiologists had been trained on the grading methods and were blinded to the macroscopic analysis results. On the 3D-FS-SPDR, the cartilage thickness of 3 parts (anterior, middle and posterior) of the medial and lateral femoral condyle was evaluated and the mean value was then used to eradicate any discrepancies.

Tissue preparation. The rabbits were sacrificed by marginal venous air embolism after anesthesia. Tissue samples were collected $2 \mathrm{~cm}$ distal and proximal to the knee and fixed in $10 \%$ neutral buffered formalin $\left(4^{\circ} \mathrm{C}\right)$ for $24 \mathrm{~h}$. After decalcification in $10 \%$ EDTA and dehydration in ethanol/xylene, samples were embedded in paraffin. Sections were cut using a microtome and stained using hematoxylin for $5 \mathrm{~min}$, eosin for $2 \mathrm{~min}(\mathrm{H} \& \mathrm{E})$ and toluidine blue for $10 \mathrm{~min}$, all at room temperature. The Mankin grading system was used to characterize the pathological changes of the cartilage.

Statistical analysis. Values are expressed as the mean \pm standard error. One-way analysis of variance was performed for multi-group comparison. The two-sample t test was utilized to analyze differences between two groups. Levene's test and the Student-Neuman-Keuls (SNK) test were utilized to analyze the Mankin score. All statistical analyses were performed using SPSS 21.0 (International Business Machines, Corp., Armonk, $\mathrm{NY}$, USA). $\mathrm{P}<0.05$ was considered to indicate a statistically significantly difference.

\section{Results}

General condition. All of the rabbits survived until the end of the experiment. The wounds healed well without any complications. Movements of the right knee were abnormal 2 weeks after the modeling surgery, particularly in group D. Neither infection of the knee joint nor patellar dislocation was revealed during tissue sampling.

\section{Imaging results}

Bone mineral density of subchondral bone. Subchondral bone loss is directly reflected by the BMD (4). The BMD in the FMC, FLC, TMC, TLC and WJK compartments of the right and the left control joints is illustrated in Fig. 1A and B. The detailed BMD data are provided in Table I. The BMD was not significantly different between the Sham and ALCT knees at week 0 after modeling $(\mathrm{P}>0.05)$ in group $\mathrm{D}$. The subchondral bone structure of the five compartments in the ACLT knees exhibited dynamic alterations after 4 and 8 weeks of OA induction; specifically a progressive decrease in BMD was observed $(\mathrm{P}<0.05)$. This indicated that the KOA rabbits exhibited increasing bone reabsorption without intervention. In comparison, the bone degeneration was significantly suppressed in the $\mathrm{ZH}$ group $(\mathrm{P}<0.05)$. In addition, the TMC compartments of ACLT knees and the Sham knees in the $\mathrm{ZH}$ group had a higher $\mathrm{BMD}(\mathrm{P}<0.05)$ at 4 and 8 weeks compared with that at week 0 , suggesting that a high dose of ZOL exerted a protective effect and even fortified the bone. In the ZM group, the BMD was stable and no significant changes were found between the baseline ( 0 week) value and the value at 4 or 8 weeks $(P>0.05)$, which indicated the subchondral bone was preserved by a medium dose of ZOL. However, in the ZL group, the FMC and TMC compartments of ACLT knees exhibited a reduced BMD at 4 weeks $(\mathrm{P}<0.05$ vs. week 0$)$. After 8 weeks, all five compartments had a decreased BMD ( $<<0.05$ vs. week 0$)$. All of the above implied that ZOL efficiently inhibited bone reabsorption in a dose-dependent manner. The doses of 250 and $50 \mu \mathrm{g} / \mathrm{kg}$ induced a favorable bone-preserving effect.

MRI scanning. MRI is able to accurately detect the progression of cartilage lesions and subchondral bone edema over an eight-week period (13). In the present study, this quantitative MRI technique was used to measure the thickness of cartilage. To reduce individual differences, cartilage thickness of ACLT joints was compared with the relevant compartment of sham joints in the same rabbit. As illustrated in Table II, in the untreated group without any ZOL intervention, the thickness of the cartilage in the FMC of the ACLT joint was identified to be significantly thinner than that in the sham knee $(\mathrm{P}<0.01)$. In the $\mathrm{ZH}$ group, the FMC cartilage in the ACLT joint was similar to that in the sham joint $(\mathrm{P}>0.05)$, whereas the cartilage of the FMC compartment of the ACLT joint in groups $\mathrm{B}$ and $\mathrm{C}$ was found to be thinner than that in the sham joint $(\mathrm{P}<0.05)$. Furthermore, regarding the FLC compartment, the cartilage in the ACLT joint in all ZOL-treated groups was no different from that in the sham joint $(\mathrm{P}>0.05)$, indicating that the high, medium and low dose of ZOL were effective to inhibit the cartilage lesions in the FLC compartment. Overall, the results implied that the high dose of ZOL exerted the highest chondroprotective effect.

Macroscopic observation. The sham knees exhibited no detectable macroscopic changes on the articular surface, which was smooth and intact with no osteophyte formation (Fig. 3). However, in the ACLT knees of the untreated group, the joint surface collapsed and became obviously flattened. Extensive cartilage erosion, and even strip and significant osteophyte formation were detected as well. With ZOL intervention, an improvement in the cartilage surface compared with that in the untreated group was seen. The ACLT joints of the ZH group displayed slight roughness and degeneration on the margin of the medial condyle. In the ZM group, the cartilage partially stripped in the medial and lateral condyle compartment. Hyperplastic fibrous connective tissue was formed as well. In the ZL group, the surface was somewhat collapsed with cartilage erosion, cartilage stripping, exposure of subchondral bone and occasional small osteophytes.

\section{Histopathological analysis}

Microscopic observation of cartilage cells with hematoxylin and eosin staining. The cartilage cells of the ACLT knees in the $\mathrm{ZH}$ group were uniformly distributed, and the tidemark was integral. The matrix was dyed evenly without any loss of dye (Fig. 4). In the ZM group, the surface layer was slightly uneven and cracks were identified in the transition zone. Occasionally, confluent cells were seen. The matrix dye was normal with uniform distribution. The degree of injury became aggravated in the ACLT knees of the ZL group. Cartilage fibrosis, cracks involving the interlayer, an irregular tidemark and non-uniform staining of the matrix were observed. The number of confluent cartilage cells was 
Table I. Detailed data of bone mineral density in each joint compartment of left and right knee $\left(\mathrm{mg} / \mathrm{cm}^{2}\right)$.

\begin{tabular}{|c|c|c|c|c|c|c|}
\hline \multirow[b]{2}{*}{ Group/knee component } & \multicolumn{3}{|c|}{ Left knee (Sham) } & \multicolumn{3}{|c|}{ Right knee (ACLT) } \\
\hline & Week 0 & Week 4 & Week 8 & Week 0 & Week 4 & Week 8 \\
\hline \multicolumn{7}{|c|}{ A, High-dose ZOL (250 $\mu \mathrm{g} / \mathrm{kg})$} \\
\hline FMC & $336 \pm 13$ & $345 \pm 11$ & $350 \pm 16$ & $346 \pm 12$ & $354 \pm 15$ & $340 \pm 14$ \\
\hline FLC & $352 \pm 17$ & $357 \pm 13$ & $364 \pm 12$ & $338 \pm 14$ & $343 \pm 17$ & $329 \pm 23$ \\
\hline TMC & $340 \pm 10$ & $343 \pm 08$ & $358 \pm 14^{\mathrm{a}}$ & $329 \pm 23$ & $351 \pm 28^{a}$ & $344 \pm 17$ \\
\hline TLC & $363 \pm 21$ & $352 \pm 25$ & $366 \pm 31$ & $351 \pm 20$ & $360 \pm 13$ & $355 \pm 18$ \\
\hline WKJ & $322 \pm 13$ & $336 \pm 16$ & $343 \pm 19^{a}$ & $316 \pm 11$ & $319 \pm 15$ & $318 \pm 09$ \\
\hline
\end{tabular}

$\mathrm{B}$, Medium-dose ZOL $(50 \mu \mathrm{g} / \mathrm{kg})$

\begin{tabular}{llllll}
\hline FMC & $373 \pm 26$ & $360 \pm 21$ & $368 \pm 18$ & $361 \pm 22$ & $370 \pm 26$ \\
FLC & $359 \pm 18$ & $367 \pm 16$ & $370 \pm 27$ & $367 \pm 25$ & $358 \pm 19$ \\
TMC & $348 \pm 13$ & $355 \pm 17$ & $362 \pm 21$ & $349 \pm 18$ & $356 \pm 22$ \\
TLC & $356 \pm 20$ & $370 \pm 33$ & $361 \pm 15$ & $370 \pm 30$ & $382 \pm 17$ \\
WKJ & $331 \pm 15$ & $340 \pm 18$ & $342 \pm 10$ & $343 \pm 12$ & $351 \pm 16$
\end{tabular}

C, Low-dose ZOL $(10 \mu \mathrm{g} / \mathrm{kg})$

\begin{tabular}{lllllll}
\hline FMC & $350 \pm 16$ & $347 \pm 13$ & $343 \pm 17$ & $362 \pm 13$ & $340 \pm 16^{\mathrm{a}}$ & $332 \pm 15^{\mathrm{a}}$ \\
FLC & $381 \pm 18$ & $395 \pm 22$ & $377 \pm 25$ & $403 \pm 30$ & $388 \pm 12$ & $376 \pm 18^{\mathrm{a}}$ \\
TMC & $359 \pm 11$ & $366 \pm 17$ & $345 \pm 14$ & $371 \pm 22$ & $353 \pm 20^{\mathrm{a}}$ & $335 \pm 30^{\mathrm{a}}$ \\
TLC & $367 \pm 23$ & $358 \pm 14$ & $353 \pm 30$ & $380 \pm 18$ & $367 \pm 15$ & $348 \pm 22^{\mathrm{a}}$ \\
WKJ & $328 \pm 08$ & $320 \pm 10$ & $315 \pm 16$ & $333 \pm 13$ & $319 \pm 11$ & $294 \pm 16^{\mathrm{a}}$ \\
\hline
\end{tabular}

D, Untreated group

\begin{tabular}{|c|c|c|c|c|c|c|}
\hline FMC & $349 \pm 15$ & $360 \pm 19$ & $353 \pm 14$ & $358 \pm 13$ & $335 \pm 18^{a}$ & $314 \pm 21^{\mathrm{a}}$ \\
\hline FLC & $383 \pm 27$ & $377 \pm 16$ & $390 \pm 23$ & $391 \pm 33$ & $369 \pm 26^{a}$ & $355 \pm 24^{\mathrm{a}}$ \\
\hline TMC & $364 \pm 18$ & $359 \pm 12$ & $355 \pm 17$ & $374 \pm 25$ & $356 \pm 23^{a}$ & $339 \pm 19^{a}$ \\
\hline TLC & $358 \pm 10$ & $364 \pm 09$ & $368 \pm 13$ & $360 \pm 11$ & $341 \pm 19^{a}$ & $325 \pm 16^{a}$ \\
\hline WKJ & $336 \pm 08$ & $341 \pm 11$ & $330 \pm 08$ & $337 \pm 14$ & $320 \pm 15^{a}$ & $295 \pm 18^{a}$ \\
\hline
\end{tabular}

Values are expressed as the mean \pm standard deviation. ${ }^{a} \mathrm{P}<0.05$ vs. baseline. ZOL, zoledronic acid; ACLT, anterior cruciate ligament transection; FMC, femoral medial condyle; FLC, femoral lateral condyle; TMC, tibial medial condyle; TLC, tibial lateral condyle; WJK, whole knee joint.

increased. In the untreated group, the observations were similar to those in the ZL group, as the cartilage displayed extension of the fibrosis range, loss of cartilage cells, partial disappearance of the tidemark and unevenly distributed matrix with severe loss of the dye.

Microscopic observation of cartilage cells with toluidine blue staining. As illustrated in Fig. 5, the femoral articular surface was smooth in ACLT knees of the ZH group and the cartilage cells were regularly arranged without any obvious loss of cells. The tibia had a flat surface with a clear gradation. A slight disorder was observed in the superficial layer of the tibial cartilage. A light loss of dye was detected in the matrix. In ACLT knees of the ZM group, the articular surface was less smooth compared with that in the ZM group. The cartilage cells were irregularly arranged with numerous vacuoles. The matrix was unevenly dyed. Vacuoles were sparse in the superficial layer of the tibial cartilage. The matrix was slightly hypochromic. In the ZL group, an irregular surface and stripped cartilage in the femur were found. The cartilage cells were unevenly dispersed with numerous vacuoles. The matrix exhibited moderate loss of dye. The tibial cartilage had a similar appearance. In ACLT knees of the untreated group, the arrangement of chondrocytes was more irregular with obvious clusters. The contour of the subchondral bone was destroyed. There was less staining in the cartilage due to the lower chondrocyte numbers.

Mankin scoring results for cartilage histopathology. The mean Mankin score of groups A (ZH), B (ZM), C (ZL) and D 
Table II. Thickness of cartilage measured by magnetic resonance imaging (mm).

\begin{tabular}{lcccc}
\hline & \multicolumn{2}{c}{ Left knee (Sham) } & & \multicolumn{2}{c}{ Right knee (ACLT) } \\
\cline { 2 - 3 } \cline { 5 - 5 } Group & FLC & FMC & FLC & FMC \\
\hline A & $1.00 \pm 0.04$ & $0.96 \pm 0.04$ & $0.96 \pm 0.08$ & $0.93 \pm 0.03$ \\
B & $1.08 \pm 0.05$ & $1.11 \pm 0.03$ & $0.98 \pm 0.03$ & $1.02 \pm 0.02^{\mathrm{a}}$ \\
C & $0.98 \pm 0.05$ & $1.08 \pm 0.05$ & $0.86 \pm 0.09$ & $0.84 \pm 0.04^{\mathrm{a}}$ \\
D & $1.06 \pm 0.05$ & $1.02 \pm 0.02$ & $0.78 \pm 0.03^{\mathrm{b}}$ & $0.80 \pm 0.03^{\mathrm{b}}$ \\
\hline
\end{tabular}

Values are expressed as the mean \pm standard deviation. ${ }^{a} \mathrm{P}<0.05,{ }^{\mathrm{b}} \mathrm{P}<0.01 \mathrm{vs}$. Sham group. Groups: A, high-dose ZOL $(250 \mu \mathrm{g} / \mathrm{kg})$; B , medium-dose ZOL (50 $\mu \mathrm{g} / \mathrm{kg}) ; \mathrm{C}$, low-dose ZOL (10 $\mu \mathrm{g} / \mathrm{kg}) ; \mathrm{D}$, untreated group. ZOL, zoledronic acid; ACLT, anterior cruciate ligament transection; FMC, femoral medial condyle; FLC, femoral lateral condyle; TMC, tibial medial condyle; TLC, tibial lateral condyle.

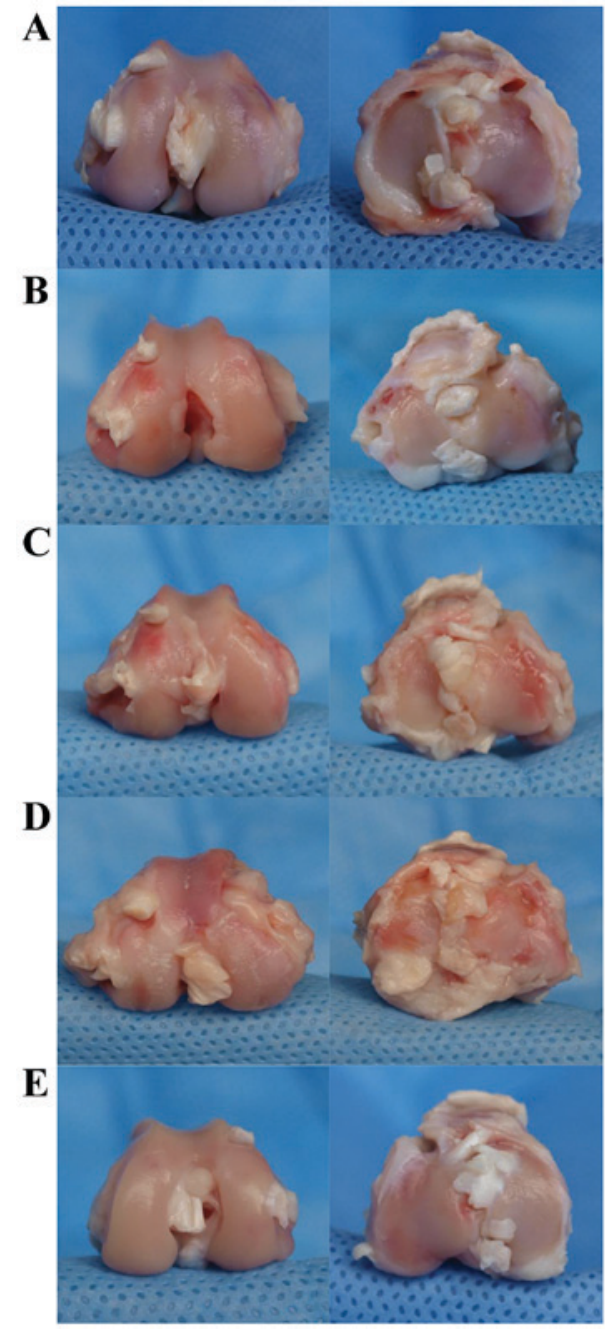

Figure 3. Representative images of the macroscopic appearance of osteoarthritic articular surface of the femur (left) and tibia (right) of the knees subjected to anterior cruciate ligament transection. (A) High-dose ZOL (250 $\mu \mathrm{g} / \mathrm{kg})$; (B) medium-dose ZOL (50 $\mu \mathrm{g} / \mathrm{kg})$; (C) low-dose ZOL $(10 \mu \mathrm{g} / \mathrm{kg})$; (D) untreated group and (E) sham-operated knee. The articular surface of the normal knee exhibited no detectable changes. Cartilage erosion as well as strip and osteophyte formation in the margin were observed in D. The surface in A had reduced damage with slight roughness and degeneration. In B and C, the defect was more severe than that in A. ZOL, zoledronic acid.

(untreated) were $3.67 \pm 0.82,4.83 \pm 1.17,6.33 \pm 1.21$ and $7.50 \pm 1.52$ (Fig. 6), respectively. Based on Mankin scores, samples may be classified as 4 different stages of OA: Nearly normal (0-2), early OA $(2<,<6)$, moderate OA $(6<,<10)$ and late OA $(10<$, $<14)(14-16)$. According to this Groups A and B may be classified as early OA and groups $\mathrm{C}$ and D may be classified as moderate OA. Significant differences among the four groups were found by Levene's test $(\mathrm{F}=11.686 ; \mathrm{P}<0.001)$. Pairwise comparison by SNK test found that the score of groups $\mathrm{C}$ and $\mathrm{D}$ were higher than those of groups $\mathrm{A}$ and $\mathrm{B}(\mathrm{P}<0.05)$.

Microscopic observation of subchondral bone. The subchondral bone cells in ACLT knees of the ZH group were regularly arranged. The bone trabecula was thick and densely distributed. No cracks were observed and the matrix was dyed evenly. In the medullary cavity, the number of blood cells decreased and the size of fat cells was less homogenous as well (Fig. 7). In the ZM group, the bone trabecula was slightly thinned with intact structure. Tiny cracks were detected locally and the dye was slightly unevenly distributed. The medullar cavity was filled with hematopoietic cells. The fat cells were homogenous in size and regularly dispersed. The subchondral bone cells in the $\mathrm{ZL}$ group were irregularly arranged. The trabeculae was thin with local breakdown. The dye was moderately unevenly distributed. Sparse hemopoietic cells and fat cells were present in the medullary cavity. In the untreated group, the subchondral bone cells were disordered. The severity of bone damage was aggravated with extensive breakdown of the trabeculae. The matrix was dyed unevenly. In the medullary cavity, the blood cells were hardly present and the shape of fat cells was neither homogenous nor intact with different size and uneven distribution.

\section{Discussion}

$\mathrm{OA}$ is a progressive rheumatic disease featuring the degeneration of articular cartilage. It is the most common rheumatic disorder and may become one of the most prevalent and costly diseases (17). The bone reduction in the OA model may be caused by post-operative synovitis with an accompanying increase in blood flow (18), an increased turnover rate (19) or by disuse of the knee after ACLT (9). In patients after ACL rupture, the BMD in the injured knee was found to be lower than that in the healthy contralateral knee (20). Articular cartilage and synovial inflammation have been the primary targets of pharmaceutical therapies for decades to attenuate joint degeneration $(21,22)$. However, the role of 


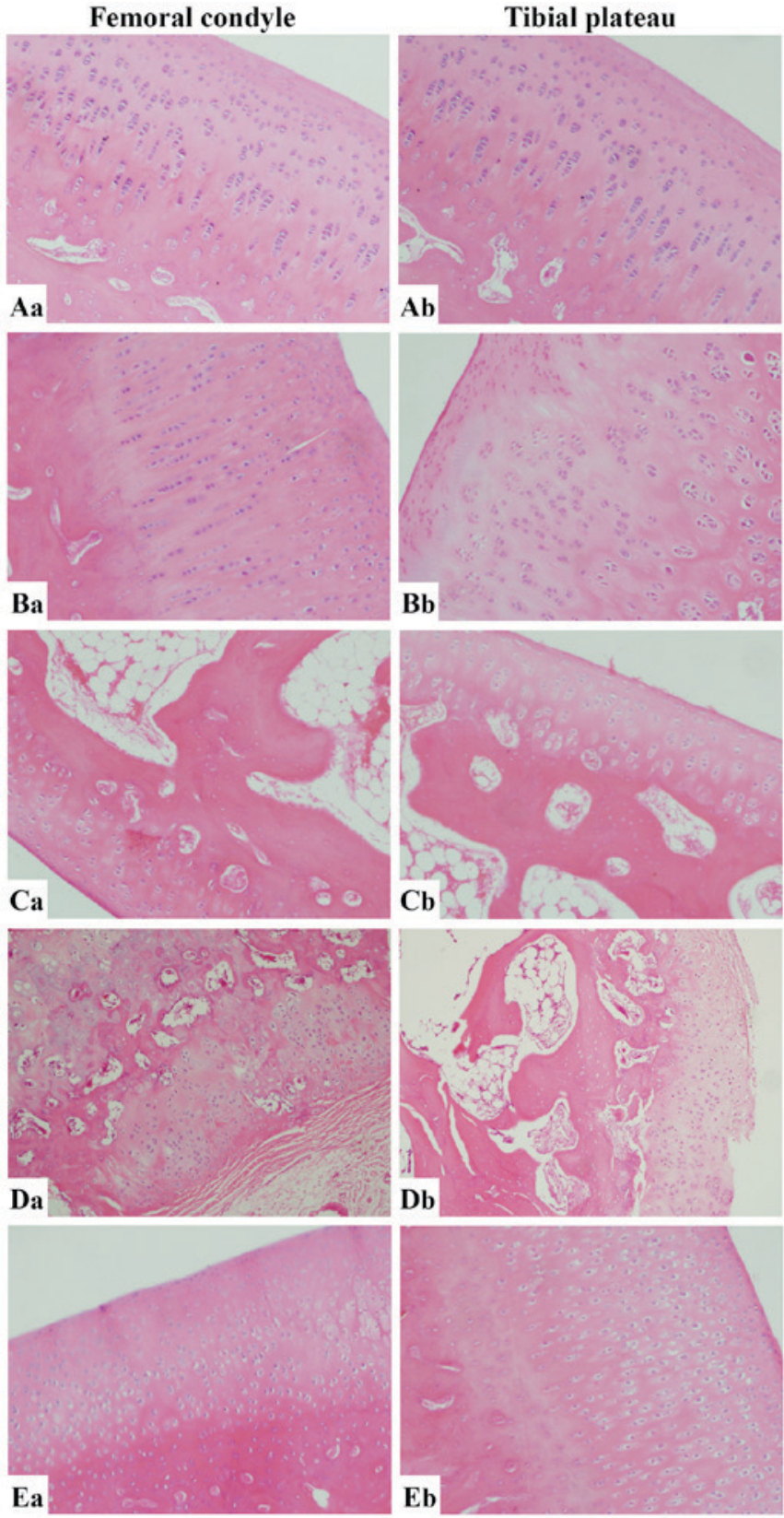

Figure 4. Cartilage cells of femoral condyle and tibial plateau from normal knees and knees subjected to anterior cruciate ligament transection (hematoxylin and eosin staining; magnification, x100). (A) High-dose ZOL $(250 \mu \mathrm{g} / \mathrm{kg})$; (B) medium-dose ZOL (50 $\mu \mathrm{g} / \mathrm{kg})$; (C) low-dose ZOL $(10 \mu \mathrm{g} / \mathrm{kg})$; (D) untreated group and (E) sham-operated knee. In D without ZOL intervention, the arrangement of chondrocytes was more irregular and their number was lower compared with those in the joints in A and B. Furthermore, the articular cartilage layer was damaged and clear boundaries between cartilage layers were lost. ZOL, zoledronic acid.

subchondral bone has been largely ignored. An increasing number of studies supported that the subchondral bone has an important role in the pathogenesis of OA. Since ZOL is a potent inhibitor of osteoclastic bone reabsorption, it may be a promising drug for ZOL reducing articular cartilage damage in OA by preventing periarticular bone loss and preserving subchondral architecture. In the present study, the ACLT joint had more periarticular bone loss than the normal joint, while ZOL treatment maintained bone density in the ACLT joint at a similar level to that of the normal knee. The subchondral
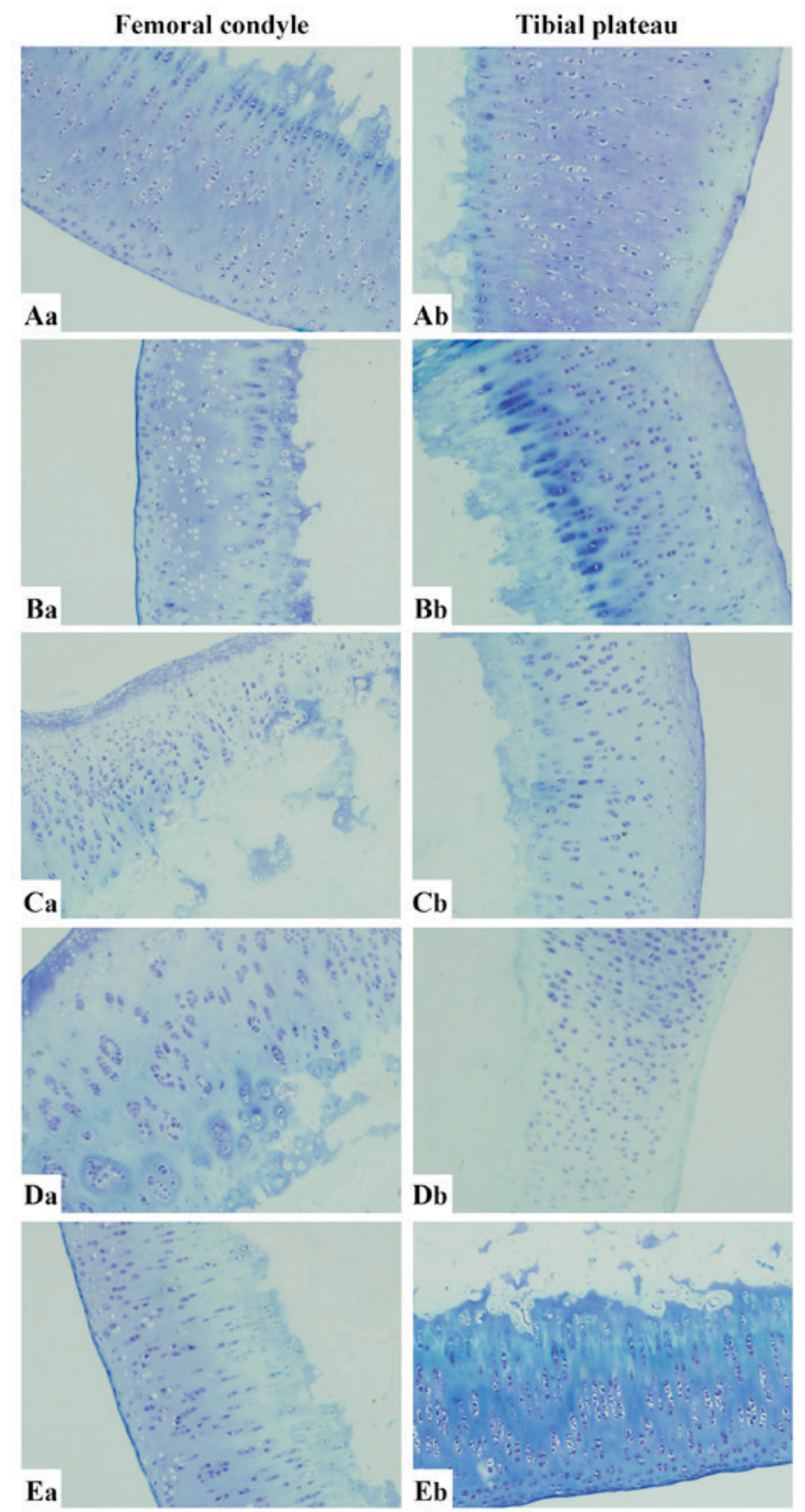

Figure 5. Cartilage cells of femoral condyle and tibial plateau from normal knees and knees subjected to anterior cruciate ligament transection (toluidine blue staining; magnification, x100). (A) High-dose ZOL (250 $\mu \mathrm{g} / \mathrm{kg})$; (B) medium-dose ZOL (50 $\mu \mathrm{g} / \mathrm{kg}$ ); (C) low-dose ZOL (10 $\mu \mathrm{g} / \mathrm{kg})$; (D) untreated group and (E) sham-operated knee. In D, the arrangement of chondrocytes was irregular with clusters. The contour of the subchondral bone was destroyed. The matrix was severely hypochromic. In comparison, in A and B, the articular surface was smoother and the cartilage cells were regularly arranged without any obvious loss of cells. ZOL, zoledronic acid.

bone in the ACLT joint was severely damaged and the bone trabeculae featured extensive breakdown. However, in the $\mathrm{ZH}$ group, the normal microstructure of the joint was preserved. These results supported the hypothesis that ZOL is a potent and promising agent to inhibit OA-associated bone reabsorption and remodeling. The use of MRI to assess the cartilage thickness and DXA to invest the BMD convincingly supported these conclusions.

Assessment of radiological joint space narrowing by X-ray radiography is the 'gold standard' for assessing OA, while 


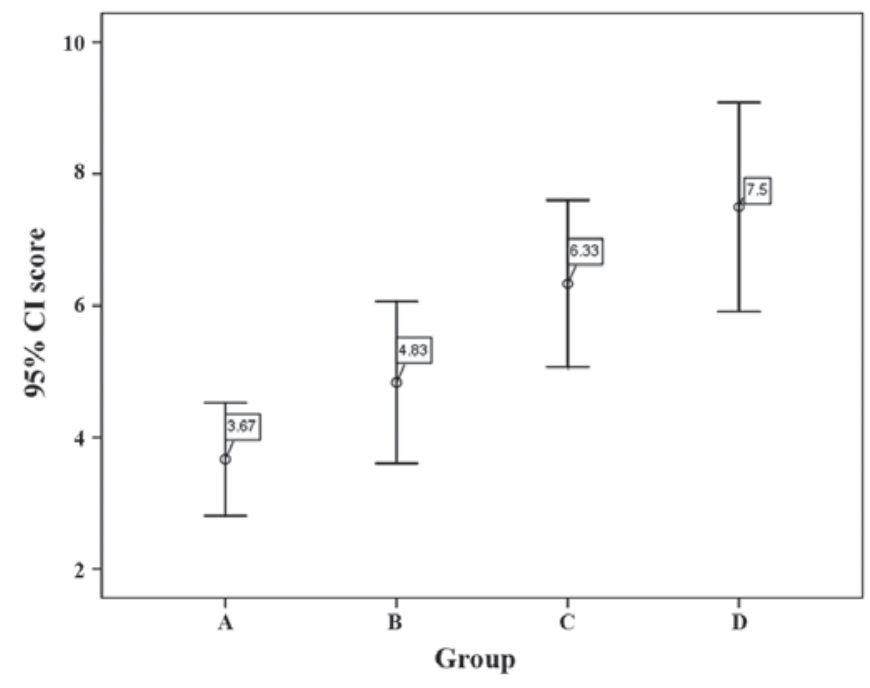

Figure 6. Mankin scores of the (A) high-dose ZOL $(250 \mu \mathrm{g} / \mathrm{kg})$, (B) mediumdose ZOL $(50 \mu \mathrm{g} / \mathrm{kg})$, (C) low-dose ZOL (10 $\mu \mathrm{g} / \mathrm{kg})$ and (D) untreated groups. ZOL, zoledronic acid.
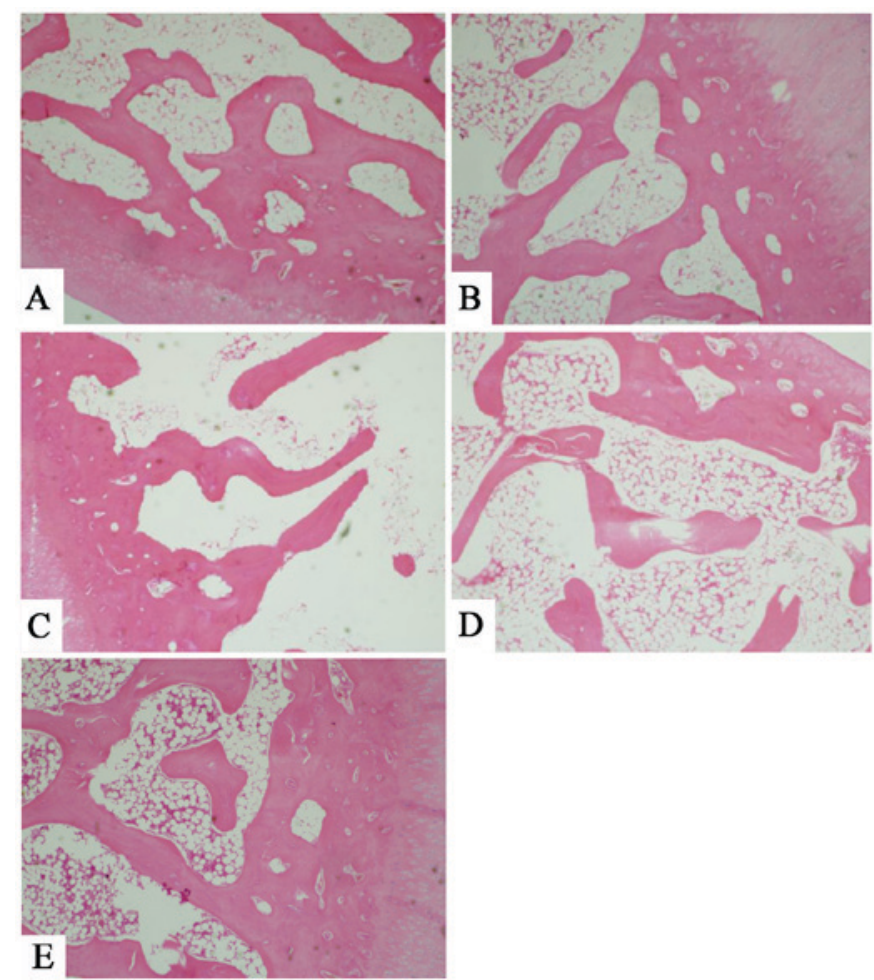

Figure 7. Microscopic observation of subchondral bone of femur (hematoxylin and eosin staining; magnification, x40). (A) High-dose ZOL (250 $\mu \mathrm{g} / \mathrm{kg})$; (B) medium-dose ZOL (50 $\mu \mathrm{g} / \mathrm{kg})$; (C) low-dose ZOL $(10 \mu \mathrm{g} / \mathrm{kg}) ;$ (D) untreated group and (E) sham-operated knee. In D, the subchondral bone cells were disordered and the matrix was dyed unevenly. The bone trabecula was sparse with obvious breakdown. The lesion was reduced in A, B and C. Particularly in A, cells were more regularly arranged compared with those in D. The bone trabecula was thick and densely distributed. No cracks were observed and the matrix was dyed evenly. ZOL, zoledronic acid.

there is currently no well-established imaging modality to visualize and quantify changes in chondral and subchondral tissue (13). MRI, with superior soft tissue contrast, is the best technique available for the assessment of normal articular cartilage and cartilage lesions (23). It makes non-invasive observation of cartilage possible. The assessment of structural changes by MRI had been previously investigated in a canine model of ACLT-induced OA (24) and in a guinea pig model of spontaneous OA (25). However, the changes in chondral and subchondral structures in a rabbit model of ACLT-induced OA have remained to be thoroughly investigated (13). The present study used 3D-FS-SPGR and T2 mapping techniques. 3D-FS-SPGR mainly focused on the morphology, particularly the detection of cartilage defects, while T2 mapping was used to quantitatively assess the cartilage $(26,27)$. Regarding to the thickness of cartilage tissue, the results for the FLC compartment were somewhat inconsistent with those in the FMC compartment. In the FLC compartment, the high, medium and low dose of ZOL were all effective to inhibit the cartilage lesion. In the FMC compartment, the cartilage was only preserved by the high dose of ZOL. The cartilages in the $\mathrm{ZM}$ and ZL groups were found to be thinner than those in the control group. The data of the FMC compartment were taken into consideration, as cartilage lesions on MRI were more severe and more commonly observed on the medial condyle (13).

The Mankin scoring system is a common histopathological evaluation method to detect articular cartilage injury. In this system, a higher the score indicates a higher the level of OA $(28,29)$. In the present study, according to their Mankin scores, the sham knees were in the normal range, and the ACLT knees in the $\mathrm{ZH}$ and $\mathrm{ZM}$ groups were categorized as early OA. The articular surface in the ZL and the untreated group were in the moderate stage of OA. This implied that reductions in the Mankin score were present after ZOL intervention. The high and medium dose of ZOL produced more favorable effects on the Mankin scores compared with the low dosage.

The cartilage histopathology evaluated by the Mankin score, cartilage thickness assessed by MRI and the macroscopic changes of subchondral bone indicated that ZOL effectively alleviated the OA-associated cartilage lesions and the dose of $250 \mu \mathrm{g} / \mathrm{kg}$ induced the most satisfactory intervention effect to protect the cartilage. The data of the present study were sufficient to confirm the hypothesis that ZOL intervened with cartilage degeneration in OA.

Of note, the present study had certain shortcomings and limitations. No blood was drawn from the rabbits due to operating difficulties, and changes in blood parameters such as bone calcium and bone turnover markers, which may have provided information on the changes on a molecular level and would have been helpful for evaluating the effect of ZOL more precisely, were not determined. This remains to be assessed in future studies.

In conclusion, ZOL prevented the development of abnormalities in subchondral bones to a large extent, and suppressed bone reabsorption in early OA. Accordingly, cartilage degeneration was reduced by ZOL. The cartilage histology, subchondral bone morphology and MRI imaging all supported the hypothesis that $\mathrm{ZOL}$ ameliorates OA-associated cartilage degradation via intervening with subchondral bone loss. Thus, the present study provided evidence supporting that ZOL presents an alternative treatment option for OA patients and may serve as an innovative therapeutic method to complement the treatment of OA. 


\section{References}

1. Felson DT and Neogi T: Osteoarthritis: Is it a disease of cartilage or of bone? Arthritis Rheum 50: 341-344, 2004.

2. Hayami T, Pickarski M, Zhuo Y, Wesolowski GA, Rodan GA and Duong LT: Characterization of articular cartilage and subchondral bone changes in the rat anterior cruciate ligament transection and meniscectomized models of osteoarthritis. Bone 38: 234-243, 2006.

3. Hayami T, Pickarski M, Wesolowski GA, McLane J, Bone A, Destefano J, Rodan GA and Duong LT: The role of subchondral bone remodeling in osteoarthritis: Reduction of cartilage degeneration and prevention of osteophyte formation by alendronate in the rat anterior cruciate ligament transection model. Arthritis Rheum 50: 1193-1206, 2004.

4. Bouchgua M, Alexander K, Carmel EN, d'Anjou MA Beauchamp G, Richard H and Laverty S: Use of routine clinical multimodality imaging in a rabbit model of osteoarthritis-part II: Bone mineral density assessment. Osteoarthritis Cartilage 17: 197-204, 2009.

5. Shirai T, Kobayashi M, Nishitani K, Satake T, Kuroki H, Nakagawa Y and Nakamura T: Chondroprotective effect of alendronate in a rabbit model of osteoarthritis. J Orthop Res 29: 1572-1577, 2011.

6. Kimmel DB: Mechanism of action, pharmacokinetic and pharmacodynamic profile, and clinical applications of nitrogen-containing bisphosphonates. J Dent Res 86: 1022-1033, 2007.

7. Bauer DC: Osteoporotic fractures: Ignorance is bliss? Am J Med 109: 338-339, 2000.

8. Black DM, Thompson DE, Bauer DC, Ensrud K, Musliner T, Hochberg MC, Nevitt MC, Suryawanshi S and Cummings SR Fracture Intervention Trial: Fracture risk reduction with alendronate in women with osteoporosis: The fracture intervention trial. FIT research group. J Clin Endocrinol Metab 85: 4118-4124, 2000

9. Ding M, Danielsen CC and Hvid I: The effects of bone remodeling inhibition by alendronate on three-dimensional microarchitecture of subchondral bone tissues in guinea pig primary osteoarthrosis. Calcif Tissue Int 82: 77-86, 2008.

10. Shuai B, Shen L, Yang Y, Ma C, Zhu R and Xu X: Assessment of the impact of zoledronic acid on ovariectomized osteoporosis model using micro-CT scanning. PLoS One 10: e0132104, 2015.

11. Yoshioka M,Coutts RD,Amiel D and Hacker SA: Characterization of a model of osteoarthritis in the rabbit knee. Osteoarthritis Cartilage 4: 87-98, 1996.

12. Mankin HJ, Dorfmon H, Lipiello L and Zarins A: Biochemical and metabolic abnormalities in articular cartilage from osteo-arthritic human hips. II. Correlation of morphology with biochemical and metabolic data. J Bone Joint Surg Am 53: 523-537, 1971.

13. Jia L, Chen J, Wang Y, Liu Y, Zhang Y and Chen W: Magnetic resonance imaging of osteophytic, chondral, and subchondral structures in a surgically-induced osteoarthritis rabbit model. PLoS One 9: e113707, 2014.

14. Zuo H, Jiang L, Qu N, Wang J, Cui X and Yao W: The biomarkers changes in serum and the correlation with quantitative MRI markers by histopathologic evaluation of the cartilage in surgically-induced osteoarthritis rabbit model. PLoS One 10: e0124717, 2015.

15. Ehrlich MG, Houle PA, Vigliani G and Mankin HJ: Correlation between articular cartilage collagenase activity and osteoarthritis. Arthritis Rheum 21: 761-766, 1978.
16. Murata M, Trahan C, Hirahashi J, Mankin HJ and Towle CA: Intracellular interleukin-1 receptor antagonist in osteoarthritis chondrocytes. Clin Orthop Relat Res: 285-295, 2003.

17. Brooks PM and March LM: New insights into osteoarthritis. Med J Aust 163: 367-369, 1995.

18. Dedrick DK, Goldstein SA, Brandt KD, O'Connor BL, Goulet RW and Albrecht M: A longitudinal study of subchondral plate and trabecular bone in cruciate-deficient dogs with osteoarthritis followed up for 54 months. Arthritis Rheum 36: 1460-1467, 1993.

19. Myers SL, Brandt KD, Burr DB, O'Connor BL and Albrecht M: Effects of a bisphosphonate on bone histomorphometry and dynamics in the canine cruciate deficiency model of osteoarthritis. J Rheumatol 26: 2645-2653, 1999.

20. van Meer BL, Waarsing JH, van Eijsden WA, Meuffels DE, van Arkel ER, Verhaar JA, Bierma-Zeinstra SM and Reijman M: Bone mineral density changes in the knee following anterior cruciate ligament rupture. Osteoarthritis Cartilage 22: 154-161, 2014.

21. Kapoor M, Martel-Pelletier J, Lajeunesse D, Pelletier JP and Fahmi H: Role of proinflammatory cytokines in the pathophysiology of osteoarthritis. Nat Rev Rheumatol 7: 33-42, 2011.

22. Martel-Pelletier J, Boileau C, Pelletier JP and Roughley PJ: Cartilage in normal and osteoarthritis conditions. Best Pract Res Clin Rheumatol 22: 351-384, 2008.

23. Bruyere O, Genant H, Kothari M, Zaim S, White D, Peterfy C, Burlet N, Richy F, Ethgen D, Montague T, et al: Longitudinal study of magnetic resonance imaging and standard X-rays to assess disease progression in osteoarthritis. Osteoarthritis Cartilage 15: 98-103, 2007.

24. Boileau C, Martel-Pelletier J, Abram F, Raynauld JP, Troncy E, D'Anjou MA, Moreau M and Pelletier JP: Magnetic resonance imaging can accurately assess the long-term progression of knee structural changes in experimental dog osteoarthritis. Ann Rheum Dis 67: 926-932,2008.

25. Tessier JJ, Bowyer J, Brownrigg NJ, Peers IS, Westwood FR, Waterton JC and Maciewicz RA: Characterisation of the guinea pig model of osteoarthritis by in vivo three-dimensional magnetic resonance imaging. Osteoarthritis Cartilage 11: 845-853, 2003.

26. Disler DG, McCauley TR, Kelman CG, Fuchs MD, Ratner LM, Wirth CR and Hospodar PP: Fat-suppressed three-dimensional spoiled gradient-echo MR imaging of hyaline cartilage defects in the knee: Comparison with standard MR imaging and arthroscopy. AJR Am J Roentgenol 167: 127-132, 1996.

27. Lee SY, Jee WH, Kim SK, Koh IJ and Kim JM: Differentiation between grade 3 and grade 4 articular cartilage defects of the knee: Fat-suppressed proton density-weighted versus fat-suppressed three-dimensional gradient-echo MRI. Acta Radiol 51: 455-461, 2010.

28. Rezende MU, Gurgel HM, Vilaça Junior PR, Kuroba RK, Lopes AS, Phillipi RZ and Hernandez AJ: Diacerhein versus glucosamine in a rat model of osteoarthritis. Clinics (Sao Paulo) 61: 461-466, 2006.

29. Armstrong S, Read R and Ghosh P: The effects of intraarticular hyaluronan on cartilage and subchondral bone changes in an ovine model of early osteoarthritis. J Rheumatol 21: 680-688, 1994. 